Monika Lisowska (D) Uniwersytet Szczeciński monika.lisowska@usz.edu.pl

\title{
Sobre la categoría gramatical de las locuciones cuantificativas elativas con estructura de sintagma nominal
}

\section{Resumen:}

En el presente artículo se exponen algunos argumentos que podrían corroborar la hipótesis de que las locuciones cuantificativas elativas con estructura de sintagma nominal son de categoría adverbial, y no adjetiva, en la cuantificación de nombres. Las observaciones presentadas se refieren al comportamiento de la preposición de en tales locuciones y a los rasgos morfosintácticos de estas en comparación con los de los adjetivos indefinidos.

Palabras clave: categoría gramatical, locuciones cuantificativas, cuantificadores nominales

\begin{abstract}
:
On the Grammatical Category of Elatively-quantifying Expressions with the Structure of a Nominal Syntagma

The article presents arguments that could support a hypothesis stating that the elatively-quantifying expressions with a nominal syntagmatic structure are adverbials, not adjectives, in the nominal quantification. The presented observations refer to the behavior of the preposition de in such expressions and to their morphosyntactic features in comparison with adjectival features of indefinite forms. Keywords: grammatical category, quantifying expressions, nominal quantifiers
\end{abstract}




\section{Presentación del problema}

El término cuantificador, relacionado con ciertas propiedades semánticas, es aplicable a palabras pertenecientes a distintas categorías gramaticales. Ahora bien, como por tradición el criterio sintáctico-funcional parece ser el más importante a la hora de determinar la clase de palabras, la clasificación gramatical de los cuantificadores españoles no siempre resulta clara, debido al problema de cómo tratar aquellas unidades que son capaces de desempeñar papeles propios de las dos categorías gramaticales siguientes: la adjetival y la adverbial ${ }^{1}$.

Este es, precisamente, el caso de ciertas locuciones cuantificativas elativas ${ }^{2}$ (de aquí en adelante: LLCCEE) con estructura de sintagma nominal, p. ej.: la tira, la mar, la leche, una burrada, una barbaridad, un montón, un porrón. Como se puede observar, se trata de las unidades compuestas por un sustantivo y el adjetivo un/a o el artículo, cuya combinación con los elementos nominales (sustantivos, adjetivos $\mathrm{y}$ adverbios) es posible solo mediante la preposición de. En el caso de estas locuciones, no hay opinión unívoca sobre su categoría gramatical, ya que todavía se pueden encontrar soluciones dicotómicas que las clasifican como adjetivales o adverbiales.

Con respecto a ello, es de interés mencionar la postura de GarcíaPage Sánchez (2008: 84), quien les confiere el estatus de policategoriales. No obstante, al utilizar el nombre de locuciones intensificadoras destaca su carácter adverbial, que manifiestan como elativos de verbos (ibidem: 99, 127). En cambio, los autores de uno de los diccionarios fraseológicos más recientes en lo que a la lengua española se refiere-Seco, Andrés, Ramos (2017)- suelen clasificar las locuciones en

1 Como observa Bosque (2007 [1989]: 128), "La naturaleza categorial de un cuantificador depende, en esa concepción, de la clase léxica del elemento sobre el que incida”.

2 El término locución cuantificativa elativa propuesto aquí hace referencia a unidades que semánticamente informan de la elación (elevación en una escala, que puede llegar, como mucho, al punto máximo de esta), siendo este un fenómeno distinto del de la superlación, donde se sobrepasa el límite de una escala (cf. Espinosa Elorza, 1998: 470-471). 
cuestión de las dos siguientes maneras: como locuciones adverbiales con el significado de 'mucho' y como sustantivos con el significado de 'una gran cantidad de algo' (cf. Seco, Andrés, Ramos, 2017, s.v. 'barbaridad', 'burrada', 'huevo', 'mar', 'montón, 'tira'). ${ }^{3}$

\section{LLCCEE con estructura de sintagma nominal en los estudios sobre los cuantificadores nominales}

Puesto que desde el punto de vista morfológico estamos ante unidades compuestas por sustantivos precedidos del artículo o del adjetivo determinativo un/a, no sorprende que las locuciones en cuestión sean objeto de estudios orientados a la sintaxis y semántica del sustantivo cuantificador, especialmente en las estructuras pseudopartitivas $N+$ $d e+N$, donde participan como primer miembro nominal. Esa unión de sustantivos también ha recibido el nombre de cuantificadores binominales ( $c f$. Verveckken, 2015).

En lo que se refiere a las clasificaciones de estas unidades, los académicos, utilizando el nombre de sustantivos cuantificativos y explicando que se trata de "(...) los sustantivos inherentemente relacionales (como montón o kilo) que cuantifican un grupo nominal sin determinante en las construcciones pseudopartitivas" (RAE y ASALE, 2009: §12.5a), los dividen en tres grupos, de los cuales el tercero sustantivos de grupo- es aquel que engloba las unidades objeto del presente estudio.

Siendo la clasificación académica de los sustantivos con carácter cuantificador una fuente de varias polémicas ( $c f$. Salazar García, 2018: 111-115), en la lingüística hispánica van apareciendo otras propuestas taxonómicas de estas unidades. Una de ellas es la de San Julián Solana, quién adopta como criterio la (in)dependencia de su carácter cuantitativo. Así, distingue los dos grupos siguientes: (a) los

3 Conviene añadir que la misma fuente trata de manera diferente las locuciones la hostia y la leche, es decir, les concede, en primer lugar, el estatus de interjecciones especializadas en expresar protesta o asombro, admitiendo que pueden funcionar también como locuciones adverbiales con el significado de 'mucho’ (cf. Seco, Andrés, Ramos, 2017, s.v. 'hostia', 'leche'). 
cuantificadores propios, que llevan las propiedades cuantitativas en su contenido léxico, por tanto, su significado cuantitativo es independiente de las circunstancias de la enunciación, y (b) los cuantificadores eventuales, que comparten la característica de ser unidades léxicamente designativas, adquiriendo el valor cuantitativo por vía metafórica (San Julián Solana, 2016b: 109-110). Según este autor, las unidades como 'la mar', 'la leche', 'un montón' o 'una barbaridad' forman parte del grupo de cuantificadores propios, puesto que en estas locuciones “(...) la asociación entre el contenido cuantitativo y el significante es estable, no dependiente del contexto" (idem, 2019: 194).

Otra de las propuestas más recientes relacionada con el objeto de nuestro estudio es la de Salazar García (2018). Esta consiste en distinguir entre cuantificadores nominales prototípicos y cuantificadores nominales no prototípicos ${ }^{4}$, quedando divididos estos últimos en dos subgrupos: cuantificadores nominales acotadores y cuantificadores nominales analógicos ${ }^{5}$. A su vez, los analógicos se agrupan en los cuantificadores mensurativos, que funcionan como tales a base de un proceso metonímico ('un dedo de tequila, 'un vaso de leche'), y los evaluativos, cuyo carácter cuantificador parece ser más bien de naturaleza metafórica ('la mar', 'una barbaridad', 'un aluvión', 'un cúmulo') (Salazar García, 2018: 127, 129).

$4 \quad$ Al proponer el término de cuantificador nominal, Salazar García quiere destacar que el factor prioritario de estas unidades es la categoría semántica de la cuantificación, siendo su pertenencia a la clase gramatical de nombre una diferencia específica (Salazar García, 2018: 117). Excluyendo los nombres de grupo y otros potenciales nombres colectivos de la categoría de cuantificación nominal, el catedrático jienense opina que los cuantificadores nominales prototípicos son los nombres numerales y los de medida (ibidem: 122-123).

5 Los acotadores "designan una porción o cuota de una determinada entidad" (Salazar García, 2018: 124), funcionando simultáneamente "como operadores de una cuantificación y como unidades con capacidad denotativa" (loc. cit.), p. ej., 'una loncha de queso' o 'una gota de leche'. A su vez, los analógicos no son capaces de expresar al mismo tiempo una cantidad y una entidad individualizable, siendo motivada su capacidad cuantificadora por una relación de analogía de carácter metafórico, metonímico o similar (ibidem: 125), p. ej.: 'una taza de café' o 'una cesta de peras'. 
Según la terminología propuesta por Salazar García, las LLCCEE con estructura de sintagma nominal pertenecerían al último de los tipos mencionados, es decir, al de cuantificadores nominales analógicos evaluativos. Se trata de un grupo formado "(...) por aquellos nombres capacitados para funcionar alternativamente, en una sincronía dada, como cuantificadores evaluativos y como nombres con valor denotativo" (Salazar García, 2018: 129). Cabe destacar que esos nombres no clasifican al restrictor, sino que su tarea es estimar "(...) en qué medida su cantidad se desvía (...) del punto medio de una escala" (loc. cit.), indicando, en la mayoría de los casos, que la cantidad es abundante o, incluso, excesiva.

Debido a la etiqueta de cuantificador nominal, la clasificación de Salazar García puede causar la impresión de que las unidades objeto de este estudio pertenecen a la categoría sustantiva. No obstante, teniendo en cuenta ciertas propiedades sintácticas de las LLCCEE con estructura de sintagma nominal, San Julián Solana en varios trabajos suyos ( $c f$. San Julián Solana, 2016a, 2016b, 2018a, 2018b, 2019) recalca que, a pesar de las apariencias, las unidades de este tipo no son realmente sustantivos. Ese autor observa que, aparte de ser capaces de modificar a adjetivos, adverbios y verbos, las unidades en cuestión demuestran su carácter no sustantivo de las maneras siguientes (San Julián Solana, 2016a: 411-420):

a) no actúan como término de concordancia "canónica" o "morfológica" al formar parte del sujeto léxico (*Falta la leche de fichas por cubrir);

b) no permiten la presencia de adyacentes adjetivos ni tampoco la de otros actualizadores (*Falta[n] la tremenda leche de fichas por cubrir, *Falta[n] esa leche de fichas por cubrir);

c) no pueden convertirse en objeto de elipsis o pronominalización (*Tenía un montón de miedo y otro de curiosidad $\left.{ }^{6}\right)$

En lo que atañe a la presencia del artículo o del adjetivo determinativo un (a) al lado de esos aparentes sustantivos, el mismo autor es de la opinión de que en estos casos ambas categorías gramaticales están

\footnotetext{
6 El ejemplo está tomado de otro trabajo de San Julián Solana (2018b: 267).
} 
fraseológicamente incorporadas, es decir, constituyen parte del significante de un signo mínimo: locución adverbial ${ }^{7}$ (San Julián Solana, 2019: 191-192).

\section{Rasgos adverbiales de las LLCCEE con estructura de sintag- ma nominal}

Según se ha podido observar, estamos ante unidades cuantificadoras procedentes de sustantivos designativos, donde el valor cuantificativo elativo, en numerosos casos, viene dado por vía metafórica, perdiéndose totalmente su referencia originaria ${ }^{8}$.

Ahora, si se quiere establecer la categoría gramatical de las unidades objeto del presente estudio, basándose en sus posibilidades sintácticas respecto a otras partes de discurso, resulta que: “(...) estas creaciones sígnicas exhiben unas pautas combinatorias que las sitúan en la categoría adverbial" (San Julián Solana, 2016b: 118), es decir, su posición sintáctica resulta ser propiamente adverbial en la mayoría de los casos:

7 Conviene destacar que con respecto a las LLCCEE que llevan incorporado el adjetivo determinativo $u n(a)$, se observa la existencia de "(...) parejas de distintos signos, aunque de significante parcialmente coincidente" (San Julián Solana, 2016b: 114), esto es, “(...) con los sustantivos montón/es, barbaridad/ es, burrada/s, porrón/es, convivirán los signos mínimos de categoría adverbial (locuciones) un montón, una barbaridad, una burrada y un porrón" (loc. cit.).

8 A favor de la motivación metafórica en la creación de las LLEECC se pronuncian Salazar García (2018: 129) y Verveckken (2015), aunque cabe subrayar que puede tratarse de una motivación un tanto opaca, con lo cual la metáfora ya no se percibe. Así lo ve San Julián Solana, quien opina que "(...) estos adverbios desustantivales no transfieren ningún rasgo semántico cualitativo a la unidad a la que caracterizan, $y$, sin dicha superposición, no tiene lugar la génesis de una 'imagen'” (San Julián Solana, 2019: 195). Además, cabe tener en cuenta que hay casos que no proceden del empleo metafórico del sustantivo. Como ejemplos, se pueden mencionar un huevo, que viene de la expresión idiomática costar ( valer) un huevo "costar mucho", y la leche o la hostia, que adquirieron el carácter cuantificador gracias a su uso como interjecciones (ibidem: 194). 
- un porrón de bueno, una barbaridad de caro, un montón de bonita, la mar de simpática, la hostia de guapa (modificadores de adjetivos)

- un montón de bien, una barbaridad de lejos (modificadores de adverbios)

- estudia inglés un montón, usábamos la bici una burrada, la alimentación ha cambiado una barbaridad (modificadores de SSVV)

Su facultad de incidir también en los sustantivos habría que relacionarla con su estructura, que exige la presencia de la preposición de para poder modificar a las categorías gramaticales principales (excepto la verbal). Esa estructura, y también sus rasgos semánticos, es lo que las hace aptas para colocarse en posición presustantival. En teoría, el hecho de poder cuantificar también a sustantivos podría considerarse como un argumento para reconocer el estatus adjetival de estas unidades, lo cual nos llevaría necesariamente a admitir que tienen una doble naturaleza categorial.

A este respecto, son de interés ciertas observaciones sobre el diferente papel de la preposición de en las estructuras partitivas -aquellas donde el primero de los sustantivos expresa una parte de objetos referidos por el segundo-y en las pseudopartitivas, en las que el segundo de los sustantivos “(...) se limita a expresar la clase designativa de lo cuantificado por el funtivo que lo precede, rechazando el valor de 'identificabilidad" " (San Julián Solana, 2018b: 262). En el grupo de estas últimas, también se encuentran esos sustantivos que han perdido su referencia originaria y funcionan como puros cuantificadores no solo de sustantivos, sino también de adjetivos y adverbios ${ }^{9}$ (ibidem: 46).

Según opina Martínez García (2008: 52), tanto en las construcciones partitivas como en las pseudopartitivas, la preposición de es fija, está únicamente al servicio de la significación cuantitativa y no cambia la categoría del segundo de los sustantivos, realizándose en

9 En opinión de San Julián Solana (2018b: 263-264), el grupo de pseudopartitivas abarca secuencias con dos estructuras radicalmente distintas: estructuras cuantitativas con complemento del nombre (una docena de estudiantes, una rebanada de pan, un grupo de planetas) y construcciones de término terciario (la tira de niños, un huevo de problemas). 
el primer caso una dependencia formal entre los dos sustantivos ${ }^{10}$ (la mayoría de los pacientes, varias de las papeletas), y, en el otro, una simple presentación del segundo sustantivo como el término regido de la relación sintáctica (un poco de agua, miles de objetos, la mar de historias). Esa autora destaca que la preposición de no ejerce su función transpositora habitual, ya que en ambos tipos de estructuras es el segundo sustantivo el núcleo (en su papel del sustantivo denotador) y el cuantificativo es su término adyacente (ibidem: 49).

De acuerdo con esa observación, Martínez García afirma que el papel de la preposición de en las estructuras partitivas consiste en adjetivar al sustantivo antepuesto, o sea, relacionar “(...) dos sustantivos mediante subordinación en un orden inverso al de otros grupos nominales que incluyen un complemento determinativo"11 (Martínez García, 2008: 54). En cambio, en las pseudopartitivas, la preposición de adverbializa el primero de los sustantivos, limitándose “(...) a ser el índice de la cuantificación, una extensión léxica del cuantificativo que la precede.” (ibidem: 51). Esta última observación surge por haberse inspirado la autora en los trabajos de José Antonio Martínez, el cual, si bien admitió la posibilidad de que algunos sustantivos sean capaces de contraer la función adverbial de "término terciario" (Martínez, 1988: 276), también opinó que esa función la realizaban respecto a “(...) un núcleo adverbial o adjetivo que comporte el morfema de 'comparación"” (ibidem). En otro trabajo suyo, el mismo autor reconoce que los términos terciarios también podían subordinarse a sustantivos (Martínez, 1994: §3.2.4.)

Aparte de todo lo expuesto hasta ahora, nos parece necesario abordar también la cuestión de la frecuente asociación de esas unidades con la categoría adjetival. Es muy posible que la inclinación a negarles el

10 La preposición de en las construcciones partitivas viene regida tanto por el sustantivo antepuesto, que sin ella no tendría valor partitivo, como por el sustantivo pospuesto, que la necesita para relacionarse con el primero (Martínez García, 2008: 52).

11 Por ejemplo, en una estructura partitiva como diez gramos de azúcar, es el elemento subrayado el que funciona como núcleo, ejerciendo los demás constituyentes la función de complemento de ese. 
estatus de adverbios cuando modifican a sustantivos se deba al tradicional trato pluricategorial de los indefinidos como 'mucho', 'poco', 'bastante', 'demasiado' ${ }^{12}$, los cuales, al igual que las unidades aquí comentadas, actúan como cuantificadores y pueden modificar a sustantivos, además de adjetivos y verbos. El carácter adjetival que se suele otorgar a esos indefinidos tiene que ver con el amplio uso del término adjetivo, relacionado con el hecho de “(...) privilegiar los dos criterios formales que caracterizan a esta clase de palabras: la concordancia con el sustantivo y su función como modificador de este." (RAE y ASALE, 2009: §13.1c). Además, se considera que el carácter adjetival de los indefinidos mencionados se deja ver también en que estos “(...) se admiten como atributos de las oraciones copulativas: (...) Mis objeciones son pocas; Los fallos descubiertos eran demasiados" (RAE y ASALE, 2009: $§ 19.1 \mathrm{k}$ ).

Seguramente, es la sensibilidad a las categorías de género y número la que hace que esos cuantificadores univerbales sean capaces de referir con mucha precisión a entidades nominales de su entorno, como p. ej.: Carlos ha escrito pocos libros, pero Pablo ha escrito muchos. Esta precisión anafórica respecto a entidades nominales la comparten, sin duda, con los adjetivos, pues estos también, gracias a sus propiedades morfológicas, pueden señalar el género y/o el número del sustantivo omitido: No hacen falta nuevos argumentos ni mejores ${ }^{13}$.

Cabe destacar que su capacidad de actuar como pronombres no se limita tan solo a los usos donde el referente es recuperable del contexto lingüístico o extralingüístico. También pueden ejercer de pronombres

12 El hecho de que otro de sus usos habituales sea el de intensificar cuantitativamente a nombres, “(...) invita a suponer que su categoría locucional no es la del adverbio sino la del adjetivo, en consonancia con los tradicionales adjetivos determinativos cuantificativos (mucho, poco, etc.)" (García-Page Sánchez, 2008: 187, n. 147). De manera parecida se pronuncian al respecto los académicos en la NGLE, cuando dicen que "La relación entre un montón y papas es similar, en efecto, a la que existe entre muchas y papas.” (RAE y ASALE, 2009: §12.5a).

13 En los ejemplos presentados, tanto los cuantificadores (muchos) como los adjetivos (mejores) modifican a una categoría nominal nula, tácita o supuesta (Bosque, 2007: 48). 
en contextos genéricos, es decir, sin la presencia de un antecedente del que se pudieran recuperar los rasgos del sustantivo omitido anafórica o deícticamente, p. ej.: Muchos solo desean lo que no pueden conseguir ${ }^{14}$.

En lo tocante a las unidades objeto de este estudio, estas no comparten -total o parcialmente- las características adjetivales de los indefinidos comentadas supra. Nuestras observaciones al respecto son las siguientes:

a) No concuerdan morfológicamente con el sustantivo modificado (*Las tiras de estudiantes), o sea, se da la neutralidad morfológica.

b) Aunque parece que la mayoría son capaces de actuar como pronombres (o, con otras palabras, demuestran capacidades fóricomostrativas $)^{15}$, en realidad el sustantivo cuantificado por estas unidades es una categoría tácita y siempre ha de poder recuperarse del contexto lingüístico para que todo el enunciado adquiera pleno sentido (San Julián Solana, 2019: 195), p. ej.: Ayer casi no recibi cartas, pero hoy me han llegado la tira [de cartas]; ¿i Qué no hubo estrellas fugaces!? Yo vi un montón [de estrellas fugaces] ${ }^{16}$. Por eso, a diferencia de los indefinidos como 'mucho' o 'poco', las LLEECC no pueden aparecer en las oraciones sin antecedente textual o extralingüístico.

c) Se puede cuestionar su capacidad para desempeñar el papel de atributos de las oraciones copulativas -“(...) diez años son la tira [de años] para un proyecto que finalmente valió la pena” (CE, 2019)- por la característica mencionada en el punto anterior: la referencia al sustantivo omitido que tiene que ser recuperado del contexto (en este caso, el que interviene en la función de sujeto).

14 El ejemplo está tomado de un trabajo de Gutiérrez Rodríguez (2018: 147), en el que la autora defiende la hipótesis de que a pesar de la ausencia del antecedente se trata de elipsis parcial, legítima solo en contextos genéricos y relacionada con una interpretación [+ humano] del sustantivo elidido.

15 Conviene destacar que la locución cuantificadora la mar de tiene la particularidad de no aceptar nunca el papel de pronombre (San Julián Solana, 2018b: 280).

16 Los ejemplos están tomados de San Julián Solana (2019: 195). 
De hecho, en este tipo de oraciones, el sustantivo, que es real atributo del predicado nominal, puede aparecer de manera explícita: Pero 80 colchones son la tira de colchones (CE, 2019).

\section{Observaciones finales}

En esta breve exposición nos hemos propuesto reflexionar sobre si es posible otorgarles una única categoría gramatical a las LLCCEE tales como la tira (de), la leche (de), una burrada (de), y otras de esta serie. Según se acaba de presentar, las unidades objeto de este estudio no comparten -total o parcialmente- ciertos rasgos adjetivales propios de los cuantificadores indefinidos univerbales, con los cuales suelen compararse al ocupar la posición presustantival. Tampoco se puede afirmar que son de categoría sustantiva cuando ejercen su función de cuantificadores respecto a verbos y adjetivos.

En principio, consideradas como adverbiales, pueden dar la impresión de que dejan de serlo al modificar a sustantivos, puesto que, según la tradición gramatical, esa posición sintáctica las convertiría en adjetivales. No obstante, queríamos destacar que hay al menos dos vías de investigación para aportar argumentos a favor de su estatus adverbial también en la posición presustantival:

a) la primera, que siguen los investigadores ovetenses, es la que consiste en analizar el papel de la preposición de en las construcciones pseudopartitivas;

b) la segunda pasa por comparar la morfosintaxis de esas unidades con la de los adjetivos determinativos: poco, mucho, demasiado, bastante.

El hecho de que sea posible que modifiquen a nombres no tiene que ser necesariamente el argumento en contra de su condición adverbial, puesto que, aunque no es muy frecuente en español, también en este idioma se dan casos de incidencia adverbial sobre grupos nominales. Según se ha intentado demostrar, el caso aquí comentado podría considerarse uno más de este tipo. 


\section{Bibliografía}

BOSQUE, I. (2007 [1989]), Las categorías gramaticales. Relaciones y diferencias, Síntesis, Madrid.

CE (2019) = DAVIES, M., Corpus del Español: NOW, [on-line] https://www. corpusdelespanol.org/, 25.07.2020.

ESPINOSA ELORZA, R. M. (1998), "Elación y superlación. Procedimientos sintagmáticos del español a lo largo de su historia", en: García Turza, C., González Bachiller, F., Mangado Martínez, J. J. (coords.), Actas del IV Congreso Internacional de Historia de la Lengua Española, La Rioja, 1-5 abril de 1997, Universidad de La Rioja, Logroño, pp. 469-480.

GARCÍA-PAGE SÁNCHEZ, M. (2008), Introducción a la fraseología española, Anthropos, Barcelona.

GUTIÉRREZ RODRÍGUEZ, E. (2018), “Interpretación genérica de sintagmas nominales con núcleo elíptico”, Borealis. An International Journal of Hispanic Linguistics, 7 (2), pp. 135-160, https://doi. org/10.7557/1.7.2.4576.

MARTÍNEZ GARCÍA, H. (2008), "Dos construcciones cuantitativas: partitiva y de término terciario", Círculo de Lingüística Aplicada a la Comunicación, 34, pp. 41-55, [on-line] http://www.ucm.es/info/circulo/no34/ martinez.pdf, 3.01.2019.

MARTÍNEZ, J. A. (1988), "Sobre una construcción del español que son dos", Verba, 15, pp. 265-287.

MARTÍNEZ, J. A. (1994), Cuestiones marginadas de gramática española, Istmo, Madrid.

REAL ACADEMIA ESPAÑOLA, ASOCIACIÓN DE ACADEMIAS DE LA LENGUA ESPAÑOLA (2009), Nueva gramática de la lengua española, Espasa, Madrid.

SALAZAR GARCÍA, V. (2018), "Cuantificadores nominales no prototípicos en español”, en: Felíu Arquiola, E. (ed.), Problemas de demarcación en morfología y sintaxis del español, Peter Lang, Berlin, pp. 111-152.

SAN JULIÁN SOLANA, J. (2016a), "El sustantivo cuantificador en la lingüística hispánica: revisión crítica y nueva propuesta”, Círculo de Lingüistica Aplicada a la Comunicación, 68, pp. 380-435, [on-line] http:// 
www.ucm.es/info/circulo/no68/sanjulian.pdf, 2.01.2019, https://doi. org/10.5209/CLAC.54535.

SAN JULIÁN SOLANA, J. (2016b), "Sobre la ambivalencia categorial de un montón y otros cuantificadores afines”, Verba Hispánica, núm. XXIV, pp. 109-123, https://doi.org/10.4312/vh.24.1.109-123.

SAN JULIÁN SOLANA, J. (2018a), “La concordancia ('ad sensum') con sustantivos cuantificadores en español”, Verba, 45, pp. 67-106, https://doi. org/10.15304/verba.45.3816.

SAN JULIÁN SOLANA, J. (2018b), "La heterogeneidad estructural de las pseudopartitivas en español", Círculo de Lingüistica Aplicada a la Comunicación, 75, pp. 261-286, [on-line] http://webs.ucm.es/info/circulo/ no75/sanjulian.pdf, 15.04.2019, https://doi.org/10.5209/CLAC.61357.

SAN JULIÁN SOLANA, J. (2019), "La obtención de cuantificadores a partir de sustantivos designativos: adaptación contextual y creación léxica", RSEL, 49, pp. 177-206, https://doi.org/10.31810/RSEL.49.8.

SECO, M., ANDRÉS, O., RAMOS, G. (2017), Diccionario fraseológico del español actual. Locuciones y modismos españoles, $2^{\mathrm{a}}$ edición, JdeJ Editores, Madrid.

VERVECKKEN, K. D. (2015), Binominal Quantifiers in Spanish. Conceptually-driven Analogy in Diachrony and Synchrony, De Gruyter, Berlin. 